\title{
CONTEXTOS DE APRENDIZAJE PARA EL DESARROLLO DEL PENSAMIENTO REFLEXIVO EN LA EDUCACIÓN SUPERIOR
}

\section{LEARNING ENVIRONMENTS FOR FACILITATING REFLECTIVE THINKING IN HIGHER EDUCATION}

\author{
Marta Sabariego Puig, Antoni Ruiz Bueno, \\ Ana Cano Hila y Angelina Sánchez Martí
}

Doctora en Filosofía y Ciencias de la Educación, Universidad de Barcelona. Profesora titular de la Universidad de Barcelona. Departamento de Métodos de Investigación y Diagnóstico en Educación, Facultad de Educación. Miembro del Grupo de Investigación en Educación Intercultural (GREDI, UB).

msabariego@ub.edu

Licenciado en Filosofía y Letras, sección Psicología. Profesor asociado de materias de metodología de investigación e investigador de la Universidad de Barcelona. Departamento de Métodos de Investigación y Diagnóstico en Educación, Facultad de Educación, Universidad de Barcelona antoniruizbueno@ub.edu 


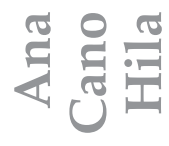

Doctora en Sociología, Universidad de Barcelona. Profesora asociada e investigadora de la Universidad de Barcelona. Miembro del grupo de investigación Creatividad, Innovación y Transformación Urbana (CRIT, UB). Departamento de Sociología, Facultad de Economía y Empresa, Universidad de Barcelona. anabelencano@ub.edu

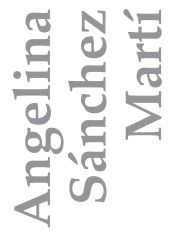

Doctora en Educación y Sociedad, Universidad de Barcelona. Profesora e investigadora postdoctoral de la Universidad Autónoma de Barcelona. Miembro del Grupo de Investigación en Migraciones, Educación e Infancia (EMIGRA, CER-Migracions). Departamento de Pedagogía Sistemática y Social, Facultad de Ciencias de la Educación, Universidad Autónoma de Barcelona.

angelina.sanchez@uab.cat

\section{RESUMEN}

Este ensayo es fruto de dos proyectos de investigación en docencia universitaria sobre el valor de las metodologías de carácter narrativo para fomentar el pensamiento reflexivo en el proceso de aprendizaje, y el desarrollo personal y profesional del alumnado universitario. El texto recoge tres apartados teóricos sobre la fundamentación conceptual del pensamiento reflexivo; las características de los contextos de aprendizaje favorables al desarrollo de esta competencia básica para el aprendizaje en la universidad; y el valor de los dispositivos narrativos diseñados con este propósito. Concluye con propuestas para contribuir a la renovación y validación de una actividad docente organizada y estructurada a este efecto en la educación superior.

Palabras clave: ambientes de aprendizaje, educación superior, narrativa, proceso del pensamiento, proceso enseñanza aprendizaje. 


\begin{abstract}
This article is the outcome of two research projects funded by the University Teaching Research grants. The projects traced the impact of narrative methodologies in reflective thinking among university students, and their impact in learning processes and their personal and professional development. The text is divided into three sections: The theoretical background on reflective thinking, the features of learning environments favouring the development of these skills, and the value of the narrative strategies to accomplish this goal. The text finishes with specific suggestions on how university teaching should be organized and structured in order to achieve the development and evaluation of reflective practice objectives so these ends might be promoted and evaluated.
\end{abstract}

Keywords: Learning environments, Higher education, Personal narratives, Teaching learning process, Thinking skills.

\title{
INTRODUCCION
}

En la educación superior se observa un énfasis creciente sobre la necesidad de la reflexión como parte integral de aprender para aprender. Se trata de formar profesionales reflexivos en su aprendizaje y en el proceso de su desarrollo competencial y profesional. Es un reto justificado si consideramos el contexto social y simbólico de las sociedades contemporáneas (Bauman, 1999; Beck, 2006; Morin, Ciurana y Mota, 2002) que sitúa el significado de la información en el núcleo de la calidad educativa para transformar las informaciones en conocimiento, comprender mejor la realidad y transformarlo en pensamiento práctico (Pérez Gómez, 2008). Las investigaciones recientes en los entornos virtuales (Chadha, 2017; Salter, Douglas y Kember, 2017), demuestran que el aprendizaje ocurre a través de un proceso igualitario en el cual los participantes generan, reflexionan y defienden sus ideas. Por lo tanto, la construcción de significado se crea a través de estos intercambios (Paul y Elder, 2012) y en un proceso de conexión entre diferentes nodos de información (Siemens, 2004). 
Este planteamiento nos lleva a pensar sobre el valor de una de las vías más importantes para construir y reconstruir significados en las actividades de enseñanza y aprendizaje, que se plantean en el espacio universitario: la reflexión. La reflexión requiere formación y hábitos, espacios adecuados de aprendizaje (Gardner, 1991), ambientes de aprendizaje (Duarte, 2003; Paniagua y Istance, 2018) e incluso una cultura de pensamiento en el aula (Ritchhart, Church y Morrison, 2014; Ritchhart, 2015). Este panorama de exigencias y retos de la sociedad basada en la información (complejidad, cambio, incertidumbre) supone un aprendizaje de las competencias desde una interpretación holística, comprensiva y más funcional (DeSeCo, 2003; Aguerrondo, 2009; Climent, 2010; Halász y Michel, 2011; Buscà, Ambròs y Burset, 2017), muy vinculado con el concepto de conocimiento práctico (Schön, 1983) para comprender e intervenir en la vida personal, social y profesional. En palabras de Pérez Gómez (2008: 71): tiene que ser necesariamente reflexivo e «incluirá habilidades mecánicas y rutinas repetitivas, pero siempre bajo la dirección de una mente reflexiva que dice cuándo, dónde y cómo utilizar estas rutinas para que sean adecuadas para entender una situación, problema o contexto». Este es el aprendizaje relevante, estratégico o el pensamiento práctico deseable en la educación superior, y que Nussbaum (2002) sitúa en una triple práctica en la enseñanza y la vida universitaria: el examen crítico de uno mismo y de la propia experiencia; la asunción de la responsabilidad social; y la empatía y reconocimiento de otras perspectivas. Este contexto básico de formación permite, además de aprender a hacer como sugiere la enseñanza por competencias, que el alumnado aprenda a ser, conocer, vivir y estar en el mundo, además de situarse, tal y como hemos podido constatar en las experiencias de innovación desarrolladas hasta la actualidad en los proyectos anteriores (Sabariego, Sánchez y Cano, 2019; Sánchez, Sabariego, Ruiz y Anglès, 2018). 


\section{ANTECEDENTES}

Este ensayo es fruto de dos proyectos de investigación ${ }^{1}$ centrados en las metodologías de carácter narrativo y (auto)biográfico (Contreras, 2011; Suárez, 2011) para fomentar el pensamiento reflexivo en lo que se aprende, en el propio proceso de aprender, y en el desarrollo competencial y profesional (Alliaud y Suárez, 2011). El interés en esta trayectoria ha permitido identificar las características de los contextos de aprendizaje favorables al desarrollo de esta competencia básica para el aprendizaje en la universidad, entendiendo el aula como un espacio para enseñar a pensar, y generar una cultura del pensamiento favorable a una enseñanza desde las habilidades reflexivas.

Los adelantos de la psicología cognitiva en las últimas décadas del siglo XX y los resultados de las investigaciones recientes, han hecho posible que el aprendizaje reflexivo se postule ya como una perspectiva educativa en Educación Superior (Brockbank y McGill, 1998, 2002) y en la actualidad es reconocido como un rasgo de calidad de la docencia universitaria, estrechamente relacionado con el desarrollo competencial del alumnado. Son escasas las definiciones de aprendizaje reflexivo disponibles en la literatura actual, pero, aun siendo diversas y variadas, sí puede destacarse un elemento común en que convergen todas ellas: comprender que se trata de un proceso en el cual intervienen de forma sustancial las habilidades reflexivas por parte de quien aprende. En este marco didáctico entendemos que el aprendizaje es el objetivo o fin que se pretende en los estudiantes y, concretamente este aprendizaje, se encuentra mediado por una reflexión activa, intencionada e instrumentada. Si bien la reflexión es un rasgo

1 Concedidos en las convocatorias de Ayudas a la Investigación en Docencia Universitaria (REDICE 14-1511 y REDICE 16-1660), financiados por el Instituto de Desarrollo Profesional-Instituto de Ciencias de la Educación de la Universitat de Barcelona. Se trata de proyectos interuniversitarios desarrollados con la participación de cinco universidades de ámbito estatal: la Universitat de Barcelona, la Universitat Autònoma de Barcelona, la Universidad de Castilla La Mancha, la Universidad de Valencia, Florida Universitaria (Unidad de Educación) y la Universidad de Burgos. 
natural del ser humano, nuestra mirada se dirige a la transformación de la reflexión ocasional en una reflexión sistemática (Domingo, 2014) para potenciar el aprendizaje profundo en la formación universitaria.

Las habilidades reflexivas aportan al aprendizaje los rasgos que le añaden valor didáctico: significatividad, funcionalidad (Ausubel, 1975) y relevancia (Pérez Gómez, 2008). Por eso podemos afirmar que el aprendizaje basado en la reflexión es el que transforma el conocimiento en aprendizaje, produciendo cambios consistentes en la estructura cognitiva del estudiante y en el cual se integran dimensiones no estrictamente cognitivas. Cuando el proceso de aprendizaje está mediado por una reflexión activa, intencionada e instrumentada se ve significativamente enriquecido al integrar más dimensiones vivenciales y emocionales: la experiencia, la intuición, las vivencias, los sentimientos, los valores, la creatividad y la singularidad de cada persona.

A partir de la diferenciación que Bateson (1973) propone sobre los tres niveles de aprendizaje, el aprendizaje reflexivo se sitúa en el nivel superior, por sus características específicas. Se trata de un aprendizaje de tercer orden que supone descubrir la capacidad de dudar de la validez de las percepciones sostenidas con anterioridad, versando el aprendizaje sobre el aprendizaje mismo. Toma el nombre de reflexivo porque es mediante la reflexión como este aprendizaje transciende sobre sí mismo. El aprendizaje reflexivo es capaz de adoptar una meta visión no solamente de los contenidos, sino también del proceso. En la medida en que el estudiante interpela al propio marco referencial (el saber construido), permite que el aprendizaje sea verdaderamente reflexivo. Estamos ante un aprendizaje de carácter activo y de estructura holística que enriquece el conocimiento, involucra las propias experiencias y el diálogo con los otros, y reconoce el contexto emocional, social y político de quien aprende.

Potenciar el aprendizaje reflexivo es propiciar en los estudiantes el desarrollo del pensamiento reflexivo (Fullana, Pallisera, Colomer, Fernández, y Pérez, 2013; González-Moreno, 2012; Phan, 2008; Romero, 2007).

Las habilidades reflexivas son importantes no solo por su papel en la adquisición de competencias, sino también en la reformulación del 
conocimiento, la práctica y la experiencia humana (Fullana et al., 2013), claves de la práctica reflexiva, entendida como el diálogo entre el pensamiento y la acción, a través del cual se aprenden nuevas y mejores habilidades, integrando la teoría y la práctica (Schön, 1983).

\section{EL PENSAMIENTO REFLEXIVO EN LA EDUCACION SUPERIOR}

John Dewey ya en 1933, en su obra How we think, definía el pensamiento reflexivo como el escrutinio de aquello que fundamenta nuestras propias creencias y sus producciones. Se trata de una actividad cognitiva que sustenta el actuar con un grado importante de originalidad, a través de la cual se realiza un cuidadoso examen de los principios que sustentan la práctica reflexiva (Romero, 2007). El pensamiento reflexivo se entiende como una competencia genérica clave para el desarrollo integral de los estudiantes universitarios (Villa y Poblete, 2007). Desde el contraste realizado de las diferentes definiciones teóricas para la docencia en este contexto (Sabariego Ed., 2018), podemos concluir que el pensamiento reflexivo es el modo de pensar que nos permite «revisar» nuestras ideas y tomar conciencia de ellas, reconocer y valorar nuestra forma de pensar. Pensar reflexivamente facilita tomar conciencia de nuestro «estilo de pensamiento», así como de los «aprendizajes realizados y en proceso», su interpretación y valoración; todos ellos elementos clave -y muy relevantes - para un ejercicio de aprendizaje sostenible, como el aprendizaje basado en competencias.

En este proceso se han identificado diversos modelos con etapas y niveles jerárquicos de reflexión (Bain, Ballantyne, Mills y Lester, 2002; Dewey, 1989; Romero, 2007; González-Moreno, 2012; Grossman, 2008; Villa y Poblete, 2007; Phan, 2008; Ryan, 2013). Los autores coinciden que la concientización constituye la dimensión de base para la acción reflexiva transformadora (Gaitán, 2007), siendo el resultado último la (re)incorporación de nuevas cualidades en el conocimiento y en las habilidades de quienes lo ejecutan. En palabras de González-Moreno (2012), «la actividad de enseñanza implica un avance, desde una actitud crítica y analítica, que permita reconstruir en detalle la experiencia para aprender» (p. 613). 
En el marco de los proyectos desarrollados, se consideraron indicadores en tres niveles jerárquicos necesarios, como base orientadora de las actividades propuestas para su desarrollo (Villa y Poblete, 2007; Ryan, 2013):

- El análisis de los procesos de pensamiento: el alumnado informa y delibera sobre un foco claro.

- Las cualidades del pensamiento del alumnado: se explicita el razonamiento, oralmente o bien por escrito, al analizar una situación o un documento, o intentar resolver un problema. Debe presentar conexiones con las propias habilidades y conocimientos previos, junto con los valores y prioridades para examinar posibilidades diferentes y considerar todas las implicaciones.

- La calidad del pensamiento crítico y transformador del alumnado. Es el nivel más alto de reflexión y el más difícil de conseguir. Los estudiantes demuestran ideas nuevas, toman decisiones específicas sobre la práctica futura: el resultado es la formación o incorporación de nuevas cualidades a los conocimientos y habilidades que ya se poseían. Cuando los estudiantes tienen la oportunidad de examinar y reflexionar sobre las propias creencias, filosofías y prácticas, es más probable que se perciban a sí mismos como agentes activos y aprendices a lo largo de la vida en su marco académico y profesional.

Los resultados obtenidos confirman el valor de elementos clave para crear contextos de aprendizaje de calidad para el desarrollo del pensamiento reflexivo en la educación superior: espacios de contraste, interacción y reflexión en el proceso de enseñanza-aprendizaje «que nos permiten llevar a cabo actos meditados de pensamiento, como tomar decisiones, argumentar y otras acciones analíticas, creativas o críticas» (Swartz, Costa, Beyer, Reagan y Kallick, 2013: 15). Otras investigaciones (Fullana et al., 2013) avalan la mayor calidad del proceso de aprendizaje y el valor del pensamiento reflexivo para esforzarse más a aprender (actividad, implicación y experimentación son principios clave de las metodologías por la escritura reflexiva), para ser más conscientes de lo que tiene sentido en el aprendizaje realizado (significatividad y funcionalidad) y para aprender de uno mismo, a partir de lo 
que ya se conoce (la escritura de la experiencia desde una perspectiva autobiográfica).

En el plano de la enseñanza, implica también un cambio de enfoque hacia nuevos espacios donde el soporte y la retroalimentación (feedback), integrados en la evaluación formativa, cobran una gran relevancia (Ion, Silva y Cano, 2013), y son claves para la optimización del pretendido desarrollo competencial y profesional en la Universidad. El papel docente es imprescindible para recrear ambientes formativos adecuados que despierten la curiosidad y la autonomía de los estudiantes en su propio proceso de aprendizaje (Medina, Jarauta, y Imbernon, 2010). La educación superior debe convertirse en un espacio de reflexión, y el pensamiento reflexivo, por lo tanto, tiene que situarse tanto en las actividades de enseñanza como en el proceso de aprendizaje.

\section{LOS CONTEXTOS DE APRENDIZAJE EN LA UNIVERSIDAD COMO ESPACIOS DE REFLEXIÓN}

El desarrollo del pensamiento exige crear espacios adecuados de aprendizaje (Gardner, 1991) que pongan en juego la reflexión e influyan en la autorregulación de este proceso por parte del alumnado (Boekaerts y Cascallar, 2006; Martínez-Fernández, 2002). A la luz de estas consideraciones, se asume que el desarrollo del pensamiento reflexivo tiene un origen social y sigue una opción metodológica desde una perspectiva socio constructivista y situada (Coll, 2012; Tejada y Ruiz, 2013; Torrano, Fuentes y Soria, 2017). Las actividades de enseñanza y de aprendizaje se conciben como experiencias sociales y emocionales para favorecer la mediación de los estudiantes consigo mismos y con los demás, conjugando la perspectiva cognitiva y emocional, la lógica narrativo-pedagógica y el aprendizaje autogestionado:

- El aula acontece un espacio donde crear situaciones que proporcionan experiencias, y el resultado de las cuales es la formación de nuevos conocimientos y habilidades en quienes las ejecuta.

- El alumnado es el protagonista en el camino de escuchar y aprender, desde la propia vivencia y experiencia, aquello que le lleva de nuevo cada materia. 
- Las estrategias narrativas a través de textos resonantes, espacios de conversación, contextos de aprendizaje fuera del aula (espacios profesionalizadores) y mediante la observación directa de la realidad, constituyen una buena opción para el aprendizaje participativo-guiado y la promoción de la reflexión en y sobre la propia práctica.

Por otra parte, el pensamiento reflexivo para el desarrollo académico o profesional implica un propósito consciente y declarado (Moon, 2006) y requiere una estructura mediatizada (Talizina, 2009), apoyada en recursos externos e internos. Las actividades de enseñanza y aprendizaje favorables al desarrollo de las habilidades reflexivas deben orientarse a que el alumnado sea consciente respecto de lo que dice y lo que hace. Por todo ello, la selección de las tareas, así como la ejecución de la estrategia y su orientación en un clima social idóneo, deben diseñarse y organizarse de manera deliberada. Estamos de acuerdo con Talizina (2009) cuando reivindica que una propuesta pedagógica con este propósito (favorable al desarrollo del pensamiento reflexivo) debe responder a la teoría de la actividad con orientación y sistematización, y a partir de unos principios que están en la base de todas las experiencias aportadas (González-Moreno, 2012): a) retroalimentación permanente en un clima social de confianza; b) autorregulación del alumnado para realizar las tareas activamente y experiencialmente; c) consignas claras sobre qué tienen que hacer y por qué deben hacerlo; y d) soporte según los requerimientos de aprendizaje.

La tarea de enseñanza, por lo tanto, va más allá de dinamizar dispositivos: exige crear condiciones, contextos con una tríada deseable en las actividades de enseñanza para el desarrollo del pensamiento reflexivo: guía, reflexión y autonomía, donde el soporte y la retroalimentación integrados en la evaluación formativa cobran una gran relevancia (Sabariego, Sánchez y Cano, 2019). También es interesante tener en cuenta el concepto de cultura de pensamiento que, en los últimos años, se ha ido consolidando progresivamente y que se entiende como «un ámbito del aula en el cual varias fuerzas (lenguaje, valores, expectativas y hábitos) operan conjuntamente para expresar y reforzar [...] el buen pensamiento» (Tishman, Perkins y Jay, 2006). En esta transformación educativa se han identificado diferentes fuerzas culturales (Ritchhart, Church 
y Morrison, 2014; Dole, 2015; Ritchhart, 2015) que, en cierto modo, influyen a la hora de crear una cultura de pensamiento y ayudar a comprender el contexto donde pueden utilizarse formas de hacer visible el pensamiento al aula: expectativas para el aprendizaje del grupo clase, oportunidades para realizar las expectativas, tiempos para pensar (al aula, en el grupo y en espacios adicionales), el modelado (por parte del docente), el lenguaje del pensamiento (condicional y que reconoce posibilidades, alternativas y perspectivas), ambiente físico, interacciones y las rutinas de pensamiento.

Desde la perspectiva de los estudiantes, un proceso de aprendizaje con actividad reflexiva requiere tres procedimientos básicos (González-Moreno, 2012; Solovieva y Quintanar, 2004): considerar el comportamiento propio (como se aprende); ser consciente de lo que se aprende, y regular el propio proceso de aprendizaje. El relato y la escritura de la experiencia a través de las estrategias narrativas (Suárez, 2011; Alliaud y Suárez, 2011), y la responsabilidad y autonomía exigidas a las modalidades de aprendizaje centrado en el estudio y trabajo autónomo del alumno (De Miguel, 2006), favorecen estos requerimientos $\mathrm{y}$, por lo tanto, se consideran actividades interesantes para promover el aprendizaje reflexivo en la Universidad. Son actividades de aprendizaje reflexivo, si bien la prioridad, de acuerdo con la línea de investigación iniciada en los proyectos anteriores, fue reforzar la producción de relatos escritos y potenciar la escritura académica como herramienta de reflexión pedagógica en la educación superior.

La escritura y la narración ayudan a construir el pensamiento (Domingo y Gómez, 2015), estableciendo la relación de este con la acción, la experiencia y los aspectos afectivos y motivacionales (autoeficacia, planteamiento de metas propias, interés por las tareas). La perspectiva narrativa y autobiográfica resulta muy interesante para fomentar la reflexión en y sobre el proceso de construcción y desarrollo competencial y profesional en la universidad (Alliaud y Suárez, 2011; Contreras, 2011; Suárez, 2011), y así lo hemos querido presentar con evidencias desde la práctica en un trabajo reciente (Sabariego, 2018). 


\section{LAS METODOLOGÍAS NARRATIVAS COMO ACTIVADORAS DEL PENSAMIENTO REFLEXIVO}

Las metodologías narrativas, propias de las ciencias sociales, se sitúan en el enfoque hermenéutico o interpretativo, y aportan al contexto social y simbólico posmoderno su énfasis en la construcción del significado y la validez desde múltiples perspectivas. Las narraciones son potencialmente ricas para producir un saber que recupera la existencia y la transforma en contenido de lo que está para venir. Además, son enormemente útiles para desarrollar la reflexividad y el pensamiento reflexivo del alumnado en su proceso de aprendizaje (Fullana et al., 2013; González-Moreno, 2012; Medina, Jarauta, e Imbernón, 2010; Pérez-Burriel, 2010; Phan, 2008; Romero, 2007).

Representan un giro epistemológico dirigido a indagar, reconstruir, documentar y comunicar los sentidos pedagógicos construidos en la experiencia educativa, y los saberes profesionales elaborados y recreados a través de la reflexión de un profesorado en permanente formación (Suárez, 2011). La escritura reflexiva exige la mediación (interna o externa) en todo aprendizaje y, afirma Contreras (2011, p. 40), es una escritura de la experiencia desde una perspectiva autobiográfica, que sitúa el alumnado en una relación pensante con lo que hace y le pasa (en el aula) para aprender con sí mismo, por sí mismo y de sí mismo. El elemento añadido radica en la posibilidad de producir ideas y reflexiones a través de lo que pasó y les pasó a los sujetos en determinadas situaciones de aprendizaje deliberativo. Con los diferentes niveles de reflexión, se facilita la unión entre el saber y el hacer (cursos de acción), así como la posibilidad de creaciones (se abren interrogantes en forma de indagaciones y (re)construcciones del conocimiento) y su trascendencia (se promueven respuestas diversas, la actividad creativa de la reflexión, abriéndose a diferentes maneras de ver la realidad).

En nuestro trabajo (Chisvert, Palomares, y Soto, 2018), el uso de los dispositivos narrativos (básicamente, relatos escritos) se confirma como útil para fomentar el pensamiento reflexivo en lo que se aprende, en el propio proceso de aprender, y en el desarrollo competencial 
y profesional del alumnado. Se trataba de poner en juego la subjetividad de los estudiantes a través del relato, indagar, reconstruir, documentar y comunicar el conocimiento construido en su experiencia de aprendizaje. La reflexión del estudiantado se incrementó mediante estrategias como el pensamiento en voz alta a la hora de resolver un problema, el análisis y el intercambio de materiales de elaboración personal, y la formulación de preguntas que el alumnado se hace, preguntas sobre los procesos y mecanismos que utilizan antes, durante y después de las tareas que realizan (Tesouro, 2005). También es interesante considerar que la reflexión no solo versó sobre la ejecución de la tarea, sino que incluía la reflexión del estudiante sobre el propio proceso de realización de las actividades propuestas (García, 2012).

En el proceso de aprendizaje, la escritura de la experiencia permitió verse, (re)pensarse, incorporarse y ponerse en juego frente aquello vivido, aquello enseñado y aquello presentado en el aula. Entre los principios de intervención para hacerlo posible, fueron especialmente relevantes:

- La introducción de una mirada autobiográfica, desde la propia experiencia, con respecto de aquello que traemos al aula sin abstraerse de lo vivido.

- La búsqueda del sentido de aquello vivido en el aula. Se trataba de permitir al alumnado ponerse en situación de novedad ante aquello vivido, pensar, dar una nueva significación a lo acontecido, preguntarse sobre el sentido de las cosas.

- El intercambio (en grupo) para «pensar con», «en presencia de»y en entornos de aprendizaje significativos. Esta mediación favorece la apertura del pensamiento, transcendiendo lo que pasa para aprender.

Las narrativas personales y experienciales han permitido situar el currículum académico en relación con la construcción de la identidad, de los significados, del saber práctico, del tiempo y de las claves cotidianas. 


\section{CONCLUSIONES}

Las evidencias obtenidas de los proyectos desarrollados permiten concluir que los estudiantes valoran positivamente las tareas diseñadas y las metodologías narrativas como activadores del pensamiento reflexivo: a) les ayudan a la gestión de la carrera dentro de su proyecto vital como futuros profesionales y como personas; b) son más conscientes de lo que tiene sentido en su aprendizaje; c) permiten un desarrollo más funcional de las competencias para comprender e intervenir en la vida personal, social y profesional, y d) se esfuerzan más a aprender y aprender de y desde uno mismo, a partir de lo que ya conocen.

También ponen de manifiesto que la tarea de enseñanza va más allá de dinamizar dispositivos: exige crear condiciones indispensables, contextos que promuevan la reflexión e influyan en la autorregulación del aprendizaje (Boekaerts y Cascallar, 2006; Martínez Fernández, 2002). El desarrollo del pensamiento reflexivo ha implicado un cambio de enfoque hacia las aulas como espacios para la reflexión con cultura de pensamiento, «lugares en donde el pensamiento del grupo, tanto individual como colectivo, se valora, se hace visible y se promueve activamente como parte de la experiencia cotidiana de todos sus miembros» (Ritchhart, Church, y Morrison, 2014).

Las experiencias desarrolladas han ilustrado diferentes contextos y situaciones de aprendizaje que conjugan la perspectiva cognitiva y emocional, la lógica narrativo-pedagógica y el aprendizaje autogestionado, alrededor de tres fuerzas culturales (Dole, 2015; Ritchhart, 2015; Ritchhart, Church, y Morrison, 2014) o tres dimensiones claves comunes para una cultura de pensamiento en la educación superior: oportunidades de aprendizaje, autorregulación y protagonismo del alumnado en el proceso de aprendizaje, y un clima social de confianza en el aula. 


\section{REFERENCIAS}

Aguerrondo, I. (2009). Complexe knowledge and education competences. Geneve, Suiza: UNESCO-IBE.

Alliaud, A., y Suárez, D. H. (2011). El saber de la experiencia. Narrativa, investigación y formación docente. Buenos Aires, Argentina: CLASCO.

Ausubel, D. (1975). «Cognitive structure and transfer». En N. Entwistle y D. Hounsell (Eds.). How Students Learn, Lancaster, Reino Unido: Institute for Reseach and Development in Post Compulsory Education.

Bauman, Z. (1999). Modernidad líquida. Buenos Aires, Argentina: Fondo de Cultura Económica.

Bateson, G. (1973). Steps Towards an Ecology of Mind. Londres, Reino Unido: Paladin.

Beck, U. (2006). Cosmopolitan vision. Cambridge, Reino Unido: Polity Press.

Boekaerts, M., y Cascallar, E. (2006). «How far have we moved toward the integration of theory and practice in self-regulation?». Educational Psychology Review, 18(3), 199-210.

Brockbank, A. y Mcgill, Y. (2002). Aprendizaje reflexivo en la Educación Superior. Madrid, España: Morata.

Buscà, F.; Ambrós, A., y Burset, S. (2017). «Bibliometric characteristics of articles on key competences indexed in ERIC from 1999 to 2013». European Journal of Teacher Education, 40(2), 144-156. Recuperado de: https: / / eric.ed.gov/ ?q=Articles\&pg=2\&id=EJ1133991

Climent, J. B. (2010). «Reflexiones sobre la educación basada en competencias». Revista Complutense de Educación, 21(1), 91-106.

Contreras, P. (2011). «Experiencia, escritura y deliberación: explorando caminos de libertad en la formación didáctica del profesorado». En A. Alliaud y D. H. Suárez (Eds.). El saber de la experiencia. Narrativa, investigación y formación docente (pp. 21-60). Buenos Aires, Argentina: CLASCO. 
Coll, C. (2005). «Constructivismo y Educación: la concepción constructivista de la enseñanza y del aprendizaje». En C. Coll, J. Palacios y A. Marchesi (Eds.). Desarrollo psicológico y Educación. 2. Psicología de la educación escolar (pp.157-186). Madrid, España: Alianza. Psicología y Educación.

Chadha, A. (2017). «Comparing student reflectiveness in online discussion forums across modes of instruction and levels of courses». Journal of Educators Online, 14(2), 1-19.

Chisvert, M. J.; Palomares, D., y Soto, D. (2018). «Las metodologías narrativas como activadoras del pensamiento reflexivo». En M. Sabariego (Ed.). El pensamiento reflexivo a través de las metodologías narrativas: experiencias de innovación en educación superior. Barcelona, España: ICE y Octaedro.

De Miguel, M. (Ed.). (2006). Modalidades de enseñanza centradas en el desarrollo de competencias. Orientaciones para promover el cambio metodológico en el EEES. Madrid, España: MEC/Universidad de Oviedo.

DeSeCo (2005). The definition and selection of key competencies. Executive summary. Recuperado de: http://deseco.ch/bfs/deseco/ en/index/03/02.parsys.78532.downloadList.94248.DownloadFile. tmp/2005.dscexecutivesummary.sp.pdf.

Dewey, J. (1989). Cómo pensamos. Cognición y desarrollo humano. Barcelona, España: Paidós.

Dole, S. F. (2017). «Creating Cultures of Thinking: The 8 Forces We Must Master to Truly Transform Our Schools». Interdisciplinary Journal of Problem-Based Learning, 11(2).

Domingo, A. y Gómez, V. (2014) La práctica reflexiva. Bases, modelos e instrumentos. Madrid, España: Narcea.

Domingo, A. (2013). Práctica reflexiva para docentes. De la reflexión ocasional a la reflexión sistemática. Saarrbrücken. Alemania: Ed. Publicia.

Duarte, D. J. (2003). «Ambientes de aprendizaje: una aproximación conceptual». Estudios Pedagógicos, 29, 97-113. 
Fullana, J., Pallisera, M., Colomer, J., Fernández, R., y Pérez, M. (2013). «Metodologías de enseñanza y aprendizaje reflexivos en la universidad. Una investigación centrada en la percepción de estudiantes de grado de la Universidad de Girona». Revista de Investigación en Educación, 11(2), 60-76.

Gaitán, C. (2007). «Paulo Freire: una pedagogía del diálogo». En: Diálogos con Freire para una pedagogía universitaria (pp. 17-29). Bogotá, Colombia: Pontificia Universidad Javeriana.

García, M. (2012). «La autorregulación académica como variable explicativa de los procesos de aprendizaje universitario». Profesorado. Revista de currículum y formación del profesorado, 16(1), 203-221.

Gardner, H. (1991). La mente no escolarizada. Barcelona, España: Paidos.

González-Moreno, C. X. (2012). «Formación del pensamiento reflexivo en estudiantes universitarios». Magis, Revista Internacional de Investigación en Educación, 4(9), 595-617.

Grossman, R. (2008). «Structures for facilitating student reflection». College Teaching, 57(1), 15-22.

Halász, G. y Michel, A. (2011). «Key competences in Europe: interpretation, policy formulation and implementation». European Journal of Education, 46(3), 289-306.

Ion, G., Silva, P., y Cano, E. (2013). «El feed-back y el feedforward en la evaluación de las competencias de estudiantes universitarios». Revista de currículum y formación profesorado, 17(2), 283-301.

Martínez Fernández, J. (2002). «Aprender: necesaria unión entre el querer, el saber y el poder». Revista de Pedagogía, 23(68), 477-494.

Medina, J. L., Jarauta, B. y Imbernon, F. (2010). La enseñanza reflexiva en la educación superior. Barcelona, España: ICE y Octaedro.

Moon, J. A. (2006). A Handbook of Reflective and Experiential Learning. Theory and Practice. Londres, Reino Unido: Routledge Falmer. 
Morin, E., Ciurana, E. R. y Mota, R. D. (2002). Educar en la era planetaria. El pensamiento complejo como método de aprendizaje en el error y la incertidumbre humana. Valladolid, España: Universidad de Valladolid, Unesco, IIPC, USAL.

Nussbaum, M. C. (2002). «Education for citizeship in an era of global connection». Studies in Philosophy and Education, 21(2), 289-303.

Paniagua, A. y Istance, D. (2018). Teachers as Designers of Learning Environments: The Importance of Innovative Pedagogies. París, Francia: OECD Publishing.

Paul, R. y Elder, L. (2012). Critical Thinking: Tools for Taking Charge of Your Learning \& Your Life. Upper Saddle River, NJ: Prentice Hall Publishing.

Pérez Gómez, A. I. (2008). «¿Competencias o pensamiento práctico? La construcción de los significados de representación y de acción». En J. Gimeno Sacristán (Ed.). Educar por competencias, ¿qué hay de nuevo? (pp. 59-102). Madrid, España: Morata.

Phan, H. P. (2008). «Metas de logro, el entorno del aula, y el pensamiento reflexivo: un marco conceptual». Revista Electrónica de Investigación Psicoeducativa, 16(6), 571-602.

Ritchhart, R.; Church, M., y Morrison, K. (2014). Hacer visible el pensamiento: Cómo promover el compromiso, la comprensión y la autonomía de los estudiantes. Barcelona, España: Paidós.

Ritchhart, R. (2015). Creating Cultures of Thinking: The 8 Forces We Must Master to Truly Transform Our Schools. San Francisco, EUA: Jossey-Bass

Romero, L. (2007). «Pensamiento reflexivo: una aproximación inicial en el ámbito de la formación de fonoaudiólogos». Revista Chilena de Fonoaudiología, 8(1), 7-14.

Ryan, M. (2013). «The pedagogical balancing act: teaching reflection in higher education». Teaching in Higher Education, 18(2), 144-151. doi: http:/ / doi.org/10.1080/13562517.2012.694104. 
Sabariego, M. (Ed.). (2018). El pensamiento reflexivo a través de las metodologías narrativas: experiencias de innovación en educación superior. Barcelona, España: ICE y Octaedro.

Sabariego, M.; Sánchez, A. y Cano, A. (2019). «El pensamiento reflexivo en la educación superior: aportaciones desde las metodologías narrativas». Revista Complutense de Educación, 30(3), 105-125.

Sánchez, A.; Sabariego, M.; Ruiz, A. y Anglès, R. (2018). «Implementation and assessment of an experiment in reflective thinking through mediated narratives to enrich higher education students' learning». Thinking Skills and Creativity, 29, 12-22. doi: 10.1016/j.tsc.2018.05.008

Salter, S., Douglas, T., y Kember, D. (2017). «Comparing face-to-face and asynchronous online communication as mechanisms for critical reflective dialogue». Educational Action Research, 25(5), 790-805.

Schön, D. (1983). The reflective practitioner. How professionals think in action. New York, EUA: Basic Books.

Siemens, G. (2004). Connectivism: A learning theory for the digital age. Recuperado de http://www.elearnspace.org/Articles/connectivism.htm

Solovieva, Y. y Quintanar, L. (2004). Métodos de intervención en la neuropsicología infantil. México: Benemérita Universidad Autónoma de Puebla.

Suárez, D. H. (2011). «Narrativas, autobiografías y formación: una presentación y algunos comentarios». Educación y Pedagogía, 61(23), $11-24$.

Swartz, R.; Costa, A.; Beyer, B.; Reagan, R. y Kallick, B. (2013). El aprendizaje basado en el pensamiento. Cómo desarrollar en los alumnos las competencias del siglo XXI. Madrid, España: SM

Talizina, N. (2009). La teoría de la actividad aplicada a la enseñanza. México: Benemérita Universidad Autónoma de Puebla.

Tejada, J., y Ruiz, C. (2016). «Evaluación de competencias profesionales en Educación Superior: retos e implicaciones». Educación XXI, 19(1), 17-39. 
Tesouro (2005). «La metacognición en la escuela: la importancia de enseñar a pensar». Educar, 35, 135-144.

Tishman, S., Perkins, D., y Jay, E. (2006). Un aula para pensar: Aprender y enseñar en una cultura de pensamiento. Buenos Aires, Argentina: Aique.

Torrano, F., Fuentes, J.L. y Soria, M. (2017). Aprendizaje autorregulado: estado de la cuestión y retos psicopedagógicos. Perfiles educativos, 39(156), 160-173.

Villa, A. y Poblete, M. (Dir.) (2008). Aprendizaje basado en competencias. Una propuesta para la evaluación de las competencias genéricas. Bilbao, España: Ediciones Mensajero. 